\title{
Entschärfte Säkularisierung - gezähmte Religiosität - (multi-)religiöse Räume als räumliche Materialisierungen der Postsäkularisierung
}

\author{
Stefanie Duttweiler \\ Berner Fachhochschule Fachbereich Soziale Arbeit, 3012 Bern, Switzerland \\ Correspondence to: Stefanie Duttweiler (stefanie.duttweiler@bfh.ch)
}

Received: 23 September 2016 - Revised: 22 April 2017 - Accepted: 19 May 2017 - Published: 12 July 2017

\begin{abstract}
Kurzfassung. Ausgangsannahme dieses Beitrags ist die architektursoziologische Prämisse, Architektur sei wesentlich an der Gestalt und Gestaltung des Sozialen beteiligt. Dementsprechend versteht der Beitrag multireligiöse Räume in nicht-religiösen Kontexten als materialisierten und verdichteten Ausdruck des religiösen und gesellschaftlichen Wandels. Durch die räumliche Verortung, die architektonische Gestaltung, die diskursiven Plausibilisierungen und Legitimierungen sowie die Nutzung der religiösen Räume wird die veränderte, postsäkulare Beziehung zwischen Religion und Gesellschaft verhandelt, transformiert und stabilisiert. Die spezifischen Herausforderungen, die sich für diese religiösen Räume ergeben, und die gestalterischen Antworten auf diese Herausforderungen entspezifizieren dabei sowohl Religion als auch Gesellschaft: Sie „entschärfen“ Säkularisierung und sie ,zähmen“ Religion.
\end{abstract}

\section{Einleitung}

Seit Mitte des 20. Jahrhunderts - und vermehrt seit den 1990er Jahren - hat sich ein neuer Raumtyp etabliert: ${ }^{1}$ Räume der Stille respektive multireligiöse Räume, die innerhalb eines bestehenden Gebäudekomplexes Raum für private Kontemplation bieten. Ihre architektonische Gestaltung verweist meist auf religiöse Räume der christlichen Religionen; in ihrer inhaltlichen Ausrichtung sind sie jedoch oft für die Nutzung durch mehrere Religionen und keine Religion offen.

\footnotetext{
${ }^{1}$ Zwischen den Weltkriegen gab es multireligiöse Räume in der US-Army, doch erst nach dem Zweiten Weltkrieg entstanden sie im zivilen Bereich (MIT-Chapel 1955, Meditationsraum im UNHauptquartier 1957, Rothko-Chapel in Houston 1971). Als erster multireligiöser Raum im deutschsprachigen Raum gilt die Flughafenkapelle in Wien (1984), erste „Räume der Stille“ entstehen in Krankenhäusern (St. Elisabeth-Hospital Gütersloh 1983). In den 1990er Jahren wurde das Kirchen-Center in der Messe Frankfurt (1990) oder der Raum der Stille im Brandenburger Tor (1994) eröffnet, doch die meisten Räume wurden erst zu Beginn des 21. Jahrhundert realisiert. Derzeit scheint ihr Ausbau allerdings zu stagnieren.
}

In den letzten Jahren wurde dieser Raumtyp zunehmend Gegenstand wissenschaftlicher Auseinandersetzungen, denn hier zeigt sich der Gestaltwandel des Religiösen in der Gegenwartsgesellschaft. Die bisherigen Forschungen widmeten sich vor allem der Funktion und Architektur dieses Raumtyps und diskutierten die gestalterischen, theologischen und sozialen Herausforderungen eines von verschiedenen Religionen gemeinsam geteilten Raumes ${ }^{2}$ (Kunst und Kirche, 2004, 2010; Gilliat-Ray, 2005; Kraft, 2007; HolsappelBrons, 2010; Brand, 2012; Nynäs, 2012; Duttweiler, 2012a, b, 2015, 2017; Compton, 2013; Beinhauer-Köhler et al., 2015; Nagel, 2015, 2016; Erne et al., 2016). Der Fokus lag mithin auf der internen Pluralität dieser Raumarrangements. Wenig Beachtung fand dagegen die Tatsache, dass sich fast alle $^{3}$ diese Räume innerhalb eines nicht-religiösen Gebäudekomplexes befinden. Sie bilden - um den instruktiven Be-

\footnotetext{
${ }^{2}$ Das Forschungsprojekt „Multi-faith-spaces. Symptoms and Agents of Change" der Universität Manchester erarbeitete eine instruktive Ausstellung, die einen hervorragenden Einblick in den internationalen Kontext liefert (online einsehbar unter: http: //cargocollective.com/wwwmulti-faith-spacesorg).

${ }^{3}$ Sehr wenige Ausnahmen bestätigen die Regel wie z.B. das „Kirchenzentrum“ in Oberhausen auf dem Gelände des Einkaufs-
} 
griff von Beinhauer-Köhler aufzugreifen - also ihrerseits mit dem Kontext ein plurales Raumarrangement (BeinhauerKöhler, 2015). Dieses spezifische architektonische Gefüge der doppelten Pluralität ist instruktiv für die Form und Funktion dieser neuen religiösen Räume, denn Raumproduktion ist immer auch mit konkreter Verortung und damit auch mit Lagebeziehungen verbunden. So stellt sich nicht nur die Frage, wie sich das Verhältnis der verschiedenen Glaubensrichtungen und Religionen unter einem Dach gestaltet, sondern auch, warum sich multireligiöse Räume in den jeweiligen funktional ausdifferenzierten räumlichen Kontexten etablieren. Wieso also gibt es in Gebäudekomplexen wie Bahnhöfen, Flughäfen, Einkaufszentren, Fußballstadien, Universitäten oder Regierungsgebäuden, die symbolischinfrastrukturelle Möglichkeitsbedingungen für distinkte soziale Systeme bereitstellen, religiöse Räume?

Im Folgenden werde ich argumentieren, dass sich in ihnen nicht nur der Gestaltwandel des Religiösen materialisiert; die Räume sind, so meine These, auch materieller Ausdruck des veränderten Verhältnisses von Religion und Gesellschaft. In ihrer räumlichen Verortung, architektonischen Gestaltung, diskursiven Plausibilisierungen und Legitimierungen sowie durch die Nutzung der religiösen Räume wird sowohl die veränderte Beziehung zwischen den Religionen als auch die veränderte Beziehung von Religion und Gesellschaft verhandelt und dabei sowohl transformiert als auch stabilisiert. Damit, so möchte dieser Artikel ausloten, sind sie Ausdruck und Motor der postsäkularen Konstellation.

\section{Religiöser Wandel und gesellschaftliche Transformation}

Auch wenn Religion in der Moderne mit Luhmann gesprochen ein Funktionssystem unter vielen ist, so war und ist für die Selbstbeschreibung der Moderne immer zentral, das Verhältnis zur Religion zu bestimmen und Säkularisierung als Signum der Moderne zu konstatieren. Doch dieses MasterNarrativ gerät zunehmend in die Krise. Zwar sind Prozesse der Deinstitutionalisierung und Enttraditionalisierung christlicher Religiosität unbestritten, umstritten ist allerdings, inwieweit sie als Säkularisierung zu deuten sind. Neben der Subjektivierung und Spiritualisierung des Religiösen bleiben viele auch den Kirchen, ihrem karitativen Engagement und ihrer Wertorientierung, verbunden. Außerdem wird Religion generell eine große Bedeutung für Kultur und Gesellschaft zugeschrieben - wie sich beispielsweise am hohen Wert, der der religiösen Erziehung zugesprochen wird, zeigt. Sie ist „als etwas durchaus (kulturell) bedeutungsvolles anerkannt, aber nur, insofern die persönliche Verpflichtung dafür delegiert werden kann“ (Knoblauch und Schnettler, 2004:9). Die Situation der Religion in Europa lässt sich damit auf die widersprüchlich erscheinenden Formeln bringen: belonging

und Vergnügungszentrums CentrO in Oberhausen sowie das „Haus der Stille“ auf dem Campus Westend der Universität Frankfurt. without believing ${ }^{4}$ (Hervieu-Léger, 2003) und believing without belonging (Davie, 1994) .

Zweifel an der Diagnose der Säkularisierung nähren sich insbesondere, wenn man über den Tellerrand von Europa sowie der christlichen Glaubensgemeinschaften hinausblickt. Doch durch Migration und kulturelle Öffnungsprozesse kommt es auch in Europa zunehmend zur Pluralisierung des Religiösen, eng damit verbunden ist ein Erstarken des Religiösen im öffentlichen Raum zu beobachten. Das zeigt sich nicht nur in einem vermehrten medialen Interesse an Religion sowie an ihrer neuen Sichtbarkeit, sondern auch im Erstarken der Fragen nach der politischen Partizipation und sozialen Verantwortung religiöser Verbände und Institutionen, die in Wohlfahrt und Politik eine Rolle spielen (Beaumont and Cloke, 2012). Dadurch verändern sich die Machtstrukturen zwischen religiösen Communities und anderen gesellschaftlichen Institutionen, was Implikationen für deren kollektive und individuelle religiöse Handlungsmacht, Identitätsbildung sowie Gemeinschafts- und Netzwerkstrukturen nach sich zieht. Casanova (1994) konstatiert daher eine Repolitisierung der religiösen Sphäre sowie eine Renormativierung der politischen und ökonomischen Sphäre.

Der Begriff der Postsäkularisierung setzt hier an: Er problematisiert (a) die empirische Gültigkeit der Säkularisierungsthese, (b) die Wiederkehr des Religiösen im öffentlichen Raum, insbesondere die vermehrte Berücksichtigung des Religiösen in politischen Debatten und Entscheidungsprozessen und den sich damit verändernden Bedingungen und Parametern des religiösen Lebens für Individuen und Gruppen (Beckford, 2012:3) und expliziert (c) in der Fassung von Jürgen Habermas (2003) eine normative Position. Dabei nennt er ,das soziologische Argument, man müsse in der Wertewelt mit kulturellen Ressourcen schonend umgehen, weil die Märkte und die administrative Macht allein die gesellschaftliche Solidarität nicht garantieren könnten, das politische Argument, dass die religiös eingestellten Bürger die gleichen staatsbürgerlichen Rechte genießen, sowie das philosophische Argument, dass ein selbstreflexiver Umgang mit den Grenzen der Aufklärung erforderlich sei“" (ReeseSchäfer, 2010:5) und daher das semantische Potential religiöser Sprache genutzt werden sollte.

Der Begriff „Postsäkularisierung“ ist mithin mehrdeutig, je nach Akzent figuriert er als gesellschaftsdiagnostischer

\footnotetext{
${ }^{4}$ Daniele Hervieu-Léger (2003) fasst darunter das Verhältnis der Europäer zur Religion als ,ein Verhältnis zu einer aus großem Abstand geteilten Erinnerung, die, auch wenn sie keinen gemeinsamen Glauben mehr impliziert, doch nach wie vor kollektive Identitätsreflexe auslöst."

${ }^{5}$ Grace Davie (1994) bezeichnet mit believing without belonging eine Verlagerung des Bezugspunkts: Statt der Bindung an religiöse Institutionen, religiöse Experten und dogmatische Wissensbestände wird nun das Individuum - seine Präferenzen und Interessen, seine „Sorge um sich“, seine Selbstverwirklichung, Entwicklung, Reinigung und Heilung - zum Maßstab und Ziel der Suche nach Sinn und Transzendenz.
} 


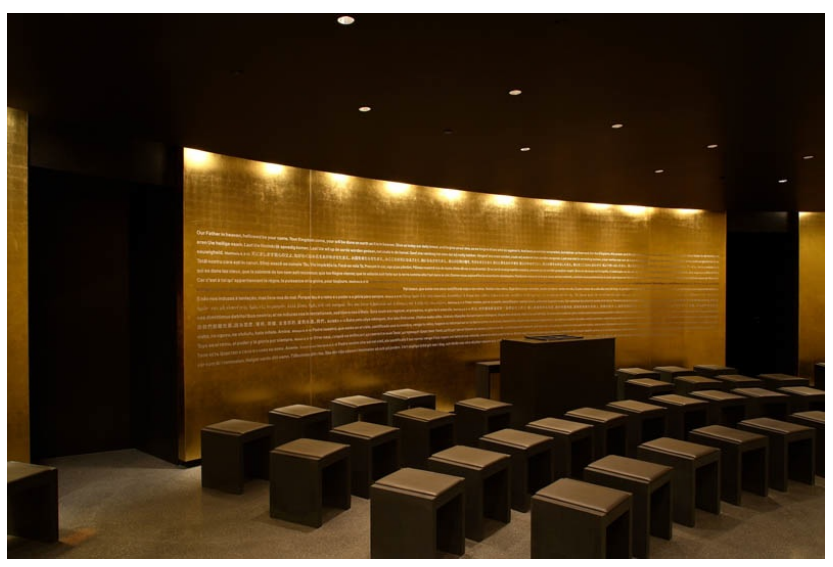

Abb. 1. Kapelle im Olympiastadion Berlin @Alfred Englert.

Begriff, der auf eine veränderte empirische Realität verweist oder als normative Position, religiöse Sinnressourcen sowie religiöse Ansprüche in liberalen Institutionen akzentuiert. Der „kleinste gemeinsame Nenner“ dieser Positionen ist die Problematisierung des Verhältnisses von Religion und gegenwärtiger Gesellschaft. Er scheint der aktuell überzeugendste Begriff zu sein, um anzuzeigen, dass die Fragen nach diesem Verhältnis auf der gesellschaftlichen Agenda stehen und dass derzeit versucht wird, Antworten darauf zu finden.

\section{Architektursoziologische Prämissen}

Die Veränderungen des Verhältnisses von Religion und Gesellschaft zeigen sich nicht zuletzt in der veränderten Landschaft des Religiösen. So ist die Präsenz nicht-christlicher Religionen ,,in der Mitte der Gesellschaft“ nicht mehr zu ignorieren (Baumann and Tunger-Zanetti, 2008; Molendijk et al., 2010) und auch die architektonischen Räume der christlichen Religionen verändern sich: Traditionelle Kirchen werden umgenutzt, geschlossen oder abgerissen und neue Räume für Religion etablieren sich. Hier setzt das Forschungsprojekt an.

Mit der Annahme, die pluralen Raumarrangements geben Hinweise auf das Verhältnis von Religion und Gesellschaft, beziehe ich mich auf die architektursoziologische Prämisse, Architektur sei wesentlich an der Gestalt und Gestaltung des Sozialen beteiligt (Delitz, 2009). Das bedeutet zum einen, dass Architektur durch Prozesse der Orts- und Raumbildung, in denen sich materielle, symbolische, imaginäre und soziale Dimensionen verschränken, Potentialitäten entfaltet, das Soziale zu formen, da sie Erwartungen und Gefühle, Verhalten, Handeln und Interaktionsabläufe wahrscheinlich macht. Zum anderen findet das Soziale in der Architektur seinen sinnlichen Ausdruck - das heißt durch die Verschränkung von materiellen und symbolischen Komponenten wird soziale Ordnung erfahrbar und so „sinnfällig“ und evident.
Möglich wird dies durch die Gestaltung von unterschiedlichen Räumen, in denen Funktionszuschreibungen und Selbstbeschreibungen der Bauherren in historisch-kulturell je spezifischer Weise materialisiert werden. Um einen solchen distinkten architektonischen Raum zu kreieren, müssen spezifische Verknüpfungen zwischen der (An-)Ordnung der materiellen Komponenten und einer symbolischen Bedeutung hergestellt werden. Wie diese Verknüpfungen gezogen werden, ist abhängig von den Relevanzkriterien (Löw, 2001), die in diskursiven und nicht-diskursiven Praktiken etabliert werden. Die konkrete Verwirklichung der architektonischen Gestalt ist dabei immer das Resultat vorhergehender Entscheidungen, die stets durch Fremdreferenzen mitbestimmt werden. So schränken statische und bauphysikalische Vorgaben die Gestaltung ebenso ein wie rechtliche Vorschriften und die Interessen der beteiligten Akteure. Eigentümer und Auftraggeber, Bauunternehmer und Fabrikanten der Materialien, Geldgeber und Politiker oder Nachbarn und Mieter vertreten dabei je persönliche, ökonomische, ethische, ästhetische, politische oder religiöse Interessen, die sich nur selten konfliktfrei miteinander vereinbaren lassen. Dementsprechend sind Größe, Lage und Gestalt eines Raumes stets das Ergebnis von Aushandlungsprozessen, ,the interests of powerful voices in the design process are etched into the artefact itself" (Gieryn, 2002:42). Das heißt nicht zuletzt, dass viele der Möglichkeiten, die gewünscht, diskutiert und geplant wurden, nicht realisiert sind. „Many possibilities become one actuality" (ebd., 43), die die anderen Möglichkeiten unsichtbar macht. In der Gestalt eines realisierten Gebäudes materialisieren sich mithin die Kräfteverhältnisse der am Prozess Beteiligten - Architektur ist somit letztlich immer auch aktueller Ausdruck dieses Verhältnisses.

Die Qualifizierung eines Raumes als religiös ist besonders anspruchsvoll, ${ }^{6}$ muss hier doch - in Abhängigkeit des je spezifischen theologischen Religions- und Raumverständnisses - mit immanenten Mitteln ein Raum hervorgebracht werden, der auf etwas Transzendentes verweist. Das ist keine geringe Herausforderung, denn es muss etwas sinnlich wahrnehmbar gemacht werden, das gerade nicht mit den Sinnen erfassbar ist, da es als prinzipiell undarstellbar und ,über-sinnlich“ " figuriert ist. Neben den theologischen Diskursen, in denen die Unterscheidung zwischen religiös und profan problematisiert und damit etabliert wird, wird der Raum durch die Verbindung verschiedener Praktiken - materieller (das Einziehen von Mauern, Gittern, Fenstern, Emporen), symbolisierender (Signifizierung durch religiöse Zeichen und Symbole) und ritueller (Weihe, Segnungen, Liturgie, Gebete, Meditationen, Kerzenopfer) - sakralisiert. Van der Leeuw (1986) argumentiert darüber hinaus, dass auch die (absichtliche) Exklusion von Außenseitern eine zentrale Rolle dabei spielt, einen sakralen Raum hervorzubringen (ebd., 52f). In diesen Prozes-

\footnotetext{
${ }^{6}$ Die eindeutige Qualifizierung als säkular ist ebenfalls problematisch, denn es existiert keine originäre Formensprache des Säkularen (Schmidt-Lux, 2017:142).
} 
sen der räumlichen Qualifizierung wird nicht zuletzt eine sakrale Atmosphäre evoziert, die den Unterschied zum profanen Raum auch leiblich-affektiv wahrnehmbar werden lässt. ${ }^{7}$ Sie soll - so der Anspruch - nicht ,kalt lassen“, sondern ,ansprechen“, „,berühren“ und „ergreifen“ und damit auf Transzendenz verweisen (Duttweiler, 2015).

Wie Martina Löw (2001) hervorgehoben hat, bedarf es neben den materiellen und symbolischen (An-)Ordnungen auch Wahrnehmungs-, Vorstellungs- und Erinnerungsprozesse der Nutzenden, um einen Raum als einen bestimmten hervorzubringen. Erst so ergibt sich eine Situationsdefinition, in die Bedeutungen und Verhaltensanweisungen sowie Stimmungen eingeschrieben sind. Die Qualifizierung eines Raumes als religiös ist somit immer auch an die einzelnen Subjekte und ihre jeweiligen Habitualisierungen gebunden - sowohl in der Errichtung als auch in der Wahrnehmung eines Raumes. Ausgehend von diesen Prämissen wird deutlich, wie schwer es ist, einen Raum zu schaffen, der für alle Besuchenden auf Religiöses verweist.

\section{Die Studie}

Im Hinblick auf die hier ausgelegte Fragestellung nach dem Verhältnis von Religion und Gesellschaft stellt sich die Frage, wie Kontext und religiöser Raum zueinander ,stehen“. (Wie) unterscheiden, überschneiden oder überlagern sie sich? (Wie) beeinflussen sie sich? Wie werden die Relationen diskursiv und architektonisch gestaltet? Um diese Fragen zu untersuchen, habe ich in der vom Schweizer Nationalfonds geförderten Studie ,Zum Gestaltwandel des Religiösen und seiner Räume" die Gestaltung, Nutzung und Bedeutungszuschreibung neuer religiöser Räume sowie das Verhältnis von religiösen und nicht-religiösen Räumen untersucht.

Gegenstand der Untersuchung waren religiöse Räume in Fußballstadien (Veltinsarena/Gelsenkirchen, AllianzArena/Frankfurt a. M., Olympiastadion/Berlin), Bahnhöfen (Zürich, Bremen, Hamburg, Berlin) und Shopping-Malls (Sihlcity-Kirche Zürich, CentrO/Oberhausen, Einkaufspassage Leipzig). Damit wurde der Fokus auf diejenigen Kontexte gelegt, in denen religiöse Räume keine Tradition aufweisen, denn gerade das Auftauchen der Räume in bislang ausschließlich säkularen Kontexten schien erklärungsbedürftig.

Ausgehend vom relationalen Raumverständnis, das Raumproduktion als Verbindung von Materialität und Bedeutung, Verortung und Lagebeziehung, Handlungen und Wahrneh-

\footnotetext{
${ }^{7}$ Die sakrale, einen Kontrast zur Umwelt erzeugende Atmosphäre wird als paradigmatisches Beispiel für Atmosphären herangezogen (bspw. Delitz, 2009; Hasse, 2014), so wie religiöse Räume als Beispiele für die formende Kraft von Architektur dienen, denn die Regelung von Körperhaltungen, Wahrnehmungen und Praktiken sowie die Institutionalisierung von Affekten trägt dazu bei, gläubige Subjekte mit zu konstituieren (Delitz, 2009:79).
}

mungen fasst, wurde ein multimethodisches Vorgehen gewählt. In einem ersten Schritt wurde mittels einer Diskursanalyse untersucht, welche Bedeutungen im öffentlichen Diskurs etablierten religiösen Räumen zugeschrieben werden. Hier zeigte sich, wie eng die religiösen mit den historischen, kulturellen und politischen Diskursen verbunden sind und dabei die Dimension des Raumes im Moment der Entselbstverständlichung des Religiösen stark aufgewertet wird. Religiöse Räume gelten als heilig, einzigartig, als Freiraum und Oase, als Hort der Barmherzigkeit und als Zeichen einer Segensdimension des Göttlichen und „stehen“ darüber hinaus auch für (Werte-)Orientierungen der ,europäischen Stadt" sowie für Identitäts- und Gemeinschaftsbildung und firmieren als individuelle und kollektive Sehnsuchts-, Erinnerungs- und Identifikationsorte. Sie werden so als Antwort auf ein als anthropologisch ausgewiesenes Bedürfnis nach Außeralltäglichkeit sowie als Bollwerk gegen moderne Desintegrationsängste figuriert (Duttweiler, 2011). Während in diesen Debatten die kulturell-historischen sowie die sozialen Aspekte eine Rolle spielen, betont - wenig überraschend - der Diskurs über Räume der Stille respektive multireligiöse Räume die Bedürfnisse nach Spiritualität, außeralltäglichen Erfahrungen oder Kontemplation und Reflexion. Auffällig ist dabei die diskursive Verhandlung der Stille. Sie bezeichnet zum einen eine bestimmte Raumgestalt, die sowohl akustisch als auch visuell von ,lärmenden“ Stimuli befreit ist. Dabei wird angenommen, die Abwesenheit von akustischem und optischem „Lärm“ versinnbildliche die NichtRepräsentierbarkeit der Transzendenz. Stille wird somit ein quasi-sakraler Charakter zugesprochen, der hinreichend offen ist und dennoch positiv besetzt werden kann. „Silence seems to be all that remains and unites in a plural and secular society“ (Holsappel-Brons, 2010:243). Damit schließt die Metapher der Stille an die Rhetorik der Neutralität an, die für einige als Königsweg der architektonischen Gestaltung gilt (Díez de Velasco, 2014) - auch wenn Neutralität weder wirklich möglich (Gilliat-Ray, 2005) noch architektonisch wünschenswert ist (Crompton, 2013). Die so entstehende ,geformte Leere“ (Romano Guardini zit. nach Kraft, 2007:33) wird dabei anthropologisch untermauert: ,Der Mensch brauche solche Orte, welche die Möglichkeit eröffnen, aus dem akustischen und optischen Lärm hinaustreten zu können, hinein in einen Raum, in welchem Schweigen herrscht und wirkliches Hören möglich wird“ (Mennekes, 2011:238). Somit wird Stille zum anderen als eine Praktik figuriert: Das Fehlen von Ablenkung erlaubt Kontemplation und fungiert so als (Vorbedingung für) religiöse Kommunikation. Diese diskursiven Figurationen wirken, so die Annahme, ihrerseits auf die Wahrnehmungen, Einstellungen und Gefühle in der Begegnung mit dem je konkreten Raum.

Stehen im Diskurs um die Räume der Stille respektive multireligiöse Räume also vor allem individuelle Bedürfnisse im Vordergrund, zeigte sich im Verlauf der Studie, dass auch die auf individuelle Praktiken ausgelegten Räume unhintergehbar verknüpft sind mit politischen, sozialen und 
ökonomischen Interessen - auch wenn das im Diskurs nicht verhandelt wird.

In einem zweiten Schritt wurden Einzelfallstudien zu den jeweiligen neuen religiösen Räumen durchgeführt. Zentraler Bestandteil waren Resonanzanalysen, in denen die Materialität und die Atmosphäre des Raumes (also die leiblichaffektiven Resonanzen) sowie ihre Verortung (also die Resonanzen zwischen dem Raum und seinem Kontext) in dichter Beschreibung erfasst wurden. Hierfür habe ich die Räume beschrieben und die durch die architektonische Ausgestaltung nahegelegten Nutzungen, körperlichen Positionierungen und Bewegungen leiblich vollzogen. Besonderen Wert wurde dabei auf die Beziehungen zwischen dem religiösen Raum und seinem Kontext gelegt. Des Weiteren wurden Experteninterviews mit den für die Räume zuständigen Geistlichen geführt, in denen Informationen über die Entstehungsbedingungen, Konfliktlinien sowie die Konzeption der Räume sowie die sie z. T. begleitenden seelsorgerischen Angebote eingeholt wurden. Wo möglich, habe ich auch beobachtend an den gottesdienstlichen Angeboten wie Andachten oder Taufen teilgenommen. Ausgewertet wurde der so entstandene Datenkorpus im Hinblick auf sich wiederholende Elemente und Charakteristika der Räume sowohl im Hinblick auf die Gestaltung, Organisation und Nutzung der Räume als auch auf die sich ergebenden Relationen zum Kontext. Im Folgenden werde ich die Ergebnisse zusammenfassen, indem ich die zentralen Neuerungen dieser pluralen Raumarrangements vorstelle: ihre Entstehung, ihre Verortung im nicht-religiösen Kontext sowie ihre (deklarierte) multireligiöse Ausrichtung.

\section{Charakteristika neuer religiöser Räume}

\subsection{Ihre Entstehung}

Neue religiöse Räume in säkularen Kontexten entstehen aus verschiedenen Motiven. Wie Francisco Díez de Velasco (2014) im Hinblick auf Flughäfen, Krankenhäuser oder Gefängnisse bemerkt, besteht dort ,, a clear need, particularly in specific scenarios such as illness or confinement, to strengthen reflection and introspection, which requires new spaces that could be described as spiritual or seclusive rather than religious in the strictest sense of the word" (ebd., 3). Für die hier untersuchten Räume in Einkaufszentren, Bahnhöfen und Fußballstadien sind diese Motive gerade nicht ausschlaggebend. Lediglich für die Bahnhofkirche in Zürich konstatiert der dortige Seelsorger, die Transiterfahrung und die Zwischenzeit des Bahnfahrens seien Gelegenheiten, sich Sinnfragen zu stellen. Und auch das für Krankenhäuser und Universitäten verbreitete Motiv, eine Gebetsgelegenheit für Muslime bereitzustellen (Gilliat-Ray, 2005), wird in den von mir untersuchten Fällen nie aufgeführt. Überraschenderweise folgte die Einrichtung der neuen religiösen Räume also keinem konkreten Bedarf, sondern konzeptionellen Überlegungen der Initiatoren.
Der Großteil der Räume geht auf die Initiative der christlichen Kirchen zurück. Die befragten Pfarrer verweisen dabei übereinstimmend auf ein neues Seelsorgekonzept: Die Kirche soll da sein, wo die Menschen sind, und sich von einer ,Komm-her-“ zu einer ,Geh-hin-Kirche“ entwickeln. Das impliziert die räumliche und konzeptionelle Annäherung an einen erweiterten Adressatenkreis. Die religiösen Angebote sollten zentral gelegen und auch für Nicht-Christen und Nicht-Religiöse einladend sein und so nicht zuletzt als „missionarische Gelegenheit“ (Pfarrer im Olympiastadion) wirken. Ein anderer (kleinerer) Teil der Räume wurde vom säkularen Kontext ins Leben gerufen. So wurde das Kirchenzentrum CentrO in Oberhausen gebaut, da das Management durch das mietfreie Überlassen eines Grundstückes an die Kirchen der gesetzlichen Vorgabe nach einer sozialen Einrichtung nachkommen konnte. Auch die Kapellen in den Fußballstadien gehen auf das Betreiben des Kontextes (d. h. der Fußballvereine $)^{8}$ zurück. In diesen Fällen herrschte zunächst Skepsis und Zurückhaltung von Seiten der Kirchen und es kam zu innerkirchlichen Auseinandersetzungen um die Konzeption und Finanzierung der religiösen Räume. Doch alle untersuchten Räume sind nun von christlichen Kirchen oder kirchennahen Institutionen als ökumenisches Projekt finanziell getragen und personell betreut - auch wenn sie als multireligiöse Räume oder Räume der Stille ausgewiesen sind.

\subsection{Verortungen - bedingte Nachbarschaften}

Das zentrale Charakteristikum dieser Räume ist ihre Integration in einen bestehenden Gebäudekomplex, hier eines Einkaufszentrums, Bahnhofs oder Fußballstadions. Auch wenn die Kontexte sehr unterschiedlich sind, ergeben sich aus dieser Verortung ähnliche konzeptionelle und gestalterische Herausforderungen. So dominiert in rechtlicher Hinsicht der Kontext; das Verhältnis ist durch die ungleichen Besitzverhältnisse und das Hausrecht geprägt. Die Kirchen sind (zahlende) Mieter, die sich im Hinblick auf Öffnungszeiten, Beschilderung und Aktivitäten an den Kontext anpassen müssen. ${ }^{9}$ Auch die Lage des religiösen Raumes kann in der Regel

\footnotetext{
${ }^{8}$ Entscheidende Motive zur Errichtung von Stadionkapellen waren die Nachahmung der Stadionkapelle im prestigeträchtigen Stadion Camp Nou in Barcelona sowie die Konkurrenz zu anderen deutschen Stadien. Dienstleistung für die Fans (Gelsenkirchen) oder ein seelsorgerisches Angebot für Spieler (Berlin) sind diesem Motivkomplex eindeutig nachgeordnet (Duttweiler, 2012a).

${ }^{9}$ So ist es der Bahnhofsmission im Berliner Hauptbahnhof nicht gestattet, eine Suppenküche anzubieten, lediglich ein Raum der Stille wird geduldet. Für die hier untersuchten Fälle konnten aber keine so drastischen Fälle berichtet werden wie der von der Jacksonville International Airport Chapel in Florida, in der Baptisten nach den Anschlägen des 11. Septembers 2001 ein tägliches Gebet in einem mietfreien zur Verfügung gestellten Raum anboten, das drei Monate später durch die Flughafenbehörde unterbunden wurde (Moodie, 2005).
} 
nicht frei bestimmt werden, was dazu führt, dass die Räume meist als Neunutzungen von Büro- oder Lagerräumen eher klein und an eher unattraktiven, unbeliebten Orten im Inneren des Gebäudekomplexes - ohne Tageslicht, oft in der Nähe der Toiletten oder des Lifts - verortet sind. Die bauliche Integration in einen anderen Gebäudekomplex impliziert auch, dass die Qualifizierung des Raumes als religiös im Außen nicht erkennbar ist; kein Turm, keine Außenwand und kein Außenraum setzen die religiösen Räume vom Kontext ab. Nicht nur die Erkennbarkeit, sondern auch die Erreichbarkeit des Raumes sind somit deutlich erschwert - sei es, dass es einer Zugangsberechtigung für den VIP-Bereich bedarf ${ }^{10}$ oder dass die Räume schwer aufzufinden sind. Die Relation zwischen den religiösen Räumen und den sie umgebenen Gebäudekomplexen erweist sich somit als bedingte Nachbarschaft: Dem Religiösen werden lediglich bestimmte Orte, Rechte und Gestaltungsmöglichkeiten „eingeräumt“.

Den Raum als religiös zu qualifizieren, gelingt im $\mathrm{Au}-$ ßen daher nur bedingt; eine minimale äußerliche Kenntlichkeit wird durch religiöse Symbole, Bibelverse oder bunte Glasfenster angestrebt. In einigen Räumen wird durch einen kleinen Vorraum - der zum Teil von ehrenamtlichen gatekeepern besetzt ist - bewusst eine Schwellenerfahrung inszeniert. Die fehlende äußere Kenntlichkeit wird jedoch in allen Räumen durch eine deutliche ästhetische und atmosphärische Markierung zwischen Außen und Innen kontrastiert. Oft ergeben sich jedoch - meist ungewollt - zahlreiche Resonanzen zwischen dem Kontext und den religiösen Räumen aufgrund der Kontinuität der architektonischen Gestaltungselemente. So ist meist Ausmaß und Materialität der Wände und Decken, die Größe und Gestaltung von Fenstern und Türen, die Ausrichtung des Raumes sowie die Ausstattung mit Klimaanlage, Steckdosen oder Notausgangsignalen durch den Kontext vorgegeben. Das reduziert nicht selten die Möglichkeit, eine sakrale Atmosphäre zu kreieren.

Diese Merkmale teilen die religiösen Räume in Fußballstadien, Einkaufszentren und Bahnhöfen mit Krankenhauskapellen oder den Räumen der Stille in Universitäten oder in Flughäfen. Um die Besonderheit dieses Samples zu verstehen, muss man daher einen Blick auf weitere Strukturmerkmale werfen, die die Kontexte - neben ihrer schieren Größe - teilen:

a. Einkaufszentren, Fußballstadien und großstädtische Bahnhöfe sind in den letzten fünfzehn Jahren räumlich und funktionell radikal nach neoliberalen Konzepten umgestaltet worden. Damit einhergehende Sicherheits- und Sauberkeitsmaßnahmen exkludieren unerwünschte Verhaltensweisen und gesellschaftliche

\footnotetext{
${ }^{10}$ Generell lässt sich beobachten: Je exklusiver der Kontext, desto exklusiver ist auch der Adressatenkreis. Der Raum der Stille im FIFA-Headquarter in Zürich ist nur Mitarbeitenden und Gästen zugänglich und auch für die Stadionkapellen muss man im Besitz einer VIP-Karte sein, ansonsten hat man nur bei Führungen oder als geladener Gast Zugang zu diesen Räumen.
}

(Rand-)Gruppen (Wehrheim, 2007). Die Nutzung der religiösen Räume ist so tendenziell auf eine urbane, mobile, konsumfreudige weiße Mittelschicht beschränkt. Das impliziert auch den potentiellen Ausschluss von Menschen mit Migrationshintergrund und damit auch mit nicht-christlicher Religionszugehörigkeit, da sie in diesen Milieus seltener vertreten sind.

b. Als multifunktionale Shopping-Malls, Sportarenen und Bahnhofswelten sind die Gebäudekomplexe nicht mehr lediglich Teil der Stadt, sondern ,simulieren“ selbst Strukturen der europäischen Stadt und sind damit paradigmatische Stätten aktueller Vergesellschaftung. Wie insbesondere in Einkaufszentren deutlich wird, kommt den religiösen Räumen damit auch die Funktion zu, diese „Stadt in der Stadt“" symbolisch zu vervollständigen (Duttweiler, 2012b).

c. Geboten und gesucht wird in den Gebäudekomplexen u. a. das besondere, nicht-alltägliche (Konsum)Erlebnis. Wesentliche Rolle spielt dabei die sogenannte brand architecture, die als Prestigearchitektur die internationale Konkurrenzfähigkeit des Gebäudes unter Beweis stellen soll.

d. Eng damit verknüpft ist die Sakralisierung des Kontextes. Sie vollzieht sich zum einen gestalterisch, indem auf Stilelemente der Sakralisierung wie Kuppeln, Hallen, Säulen, edle Materialien oder eine spezifische Lichtführung zurückgegriffen wird. Darüber hinaus werden Fußball (Klein/Schmidt-Lux, 2006) oder Shopping (Isenburg/Sellmann, 2000; Sorgo, 2008) Funktionen wie Ritualisierung, Sinnstiftung und Vergemeinschaftung oder die Produktion „heiliger“ Zeiten und Orte zugeschrieben, die den Vergleich mit Religion nahelegen.

Die architektonische „Antwort“ der religiösen Räume auf diese Kontextbedingungen ist in einigen Fällen die bewusst gestaltete „Ebenbürtigkeit“ mit dem Kontext. So sind die preisgekrönten Räume wie die von einem bekannten Schweizer Künstler mitgestaltete Sihlcity-Kirche in Zürich, dem aus reinem Onyx bestehenden Meditationsraum des FIFAHeadquarters oder die golden ausgekleidete Kapelle des Berliner Olympiastadions Teil der Prestigearchitektur. Auch konzeptionell gibt es Annäherungen an den neoliberalisierten, sakralisierten Kontext. So erweitern beispielsweise in den Stadionkappellen Taufen und Hochzeiten für FußballFans die Dienstleistung des Stadions um ein stark nachgefragtes Angebot. In anderen Fällen wird konzeptionell eher ein Kontrapunkt zum kommerziellen Angebot des Kontextes angestrebt, indem sie kostenlosen, konsum- und werbefreien Raum zum ungestörten Aufenthalt oder beraterischen und seelsorgerischen Beistand anbieten. Entkommen können sie der Verwertungslogik des Kontextes damit jedoch kaum. So dämmt beispielsweise das Kirchenzentrum im CentrO in Oberhausen die Kritik an der Shopping-Mall ein, an den 
meisten anderen Orten kümmern sich die Seelsorger um Bedürftige oder übernehmen Gespräche mit Personen, die „Ärger machen“, oder sie kümmern sich um die seelischen Belange der BesucherInnen und Mitarbeitenden. Damit tragen sie im weitesten Sinne zur „Befriedung“ des Kontextes bei, der von diesen Dienstleistungen profitiert.

Doch auch für die die Räume unterhaltenden kirchlichen Institutionen ergibt sich ein Prestigegewinn. ${ }^{11}$ Mit ihnen können sich die Kirchen als offen, modern und weltzugewandt präsentieren und zugleich die von ihnen erwartete Differenz zur Welt betonen. Sie sind - das zeigt auch ihre Nutzung (s. u.) - ,eine gute ,public relation“" für die Kirchen (Pfarrer Schlauri, Bahnhofskirche Zürich). Aus ökonomischer Perspektive erweisen sich die Räume so auch als Antwort der Institution Kirche auf die Notwendigkeit, als politischer oder wirtschaftlicher Akteur eine erfolgversprechende Marketingstrategie zu lancieren.

Die Verortung der bedingten Nachbarschaft und nicht zuletzt diese Win-win-Situation zeigt, dass die Relationen in diesen Raumarrangements vielschichtig sind: Religiöses und Profanes sind in diesen kommerziellen Kontexten, die zugleich hochgradig exklusiv und somit exkludierend sind, voneinander abgegrenzt, doch zugleich in vielfältiger Hinsicht unhintergehbar ineinander verschränkt.

\subsection{Architektonische Gestaltung - deklarierte Offenheit und realisierte Schließung}

Neben der spezifischen Verortung ist auch die innere Qualifizierung der Räume aussagekräftig, denn nicht zuletzt materialisieren sich hier immer auch die je gegenwärtigen theologischen Konzepte. Im untersuchten Sample lassen sich drei verschiedene Raumtypen unterscheiden:

1. christliche Kapellen. Die religiösen Räume in Fußballstadien sind ausschließlich für eine christliche Nutzung vorgesehen. Dementsprechend orientieren sie sich in der Namensgebung, räumlichen Ausrichtung, architektonischen Gestaltung sowie der Ausstattung mit religiösen Symbolen und Paraphernalien eng an traditionellen Kirchen- respektive Kapellenbauten und deren hohem ästhetischen Anspruch. Die religiöse Qualifizierung ergibt sich neben der sakralen Form- und Symbolsprache auch über die regelmäßig stattfindenden Kausaliengottesdienste. Offenheit ist kein erklärter Anspruch; die Kapellen bieten weder für diejenigen, die einer

\footnotetext{
${ }^{11}$ Ein Imagegewinn wird auch in anderen Kontexten erhofft, wie Alexander-Kenneth Nagel (2015) für multireligiöse Räume in konfessionellen Krankenhäusern oder Christel Beinhauer Köhler (2015) für religiöse Räume in Regierungsgebäuden herausgearbeitet haben. Letztere, so Beinhauer-Köhler, dienen vor allem der ,religionspolitische[n] Repräsentation“ (ebd., 64): ,Es scheint für die Träger oder Initiatoren als politisch geboten, ein solches Projekt zu unterstützen, da es ein positives Zeichen zur Gestaltung der Zivilgesellschaft setzt" (ebd., 72).
}

nicht-christlichen oder keiner Religion angehören, noch für diejenigen, die sich ohne rituelle Vollzüge darin aufhalten möchten, Raum.

Alle anderen untersuchten Räume sind zwar ebenfalls von kirchlichen Institutionen getragen (und heißen z. T. auch Kirchen), doch sie postulieren Offenheit auch für andere Religionen und keine. Hierin liegt eine große architektonische Herausforderung, denn es fehlen eindeutig erkennbare architektonische Codes. Soll der Raum eine multireligiöse Ausrichtung erhalten, kann man nicht auf institutionalisierte „,building types“ zurückgreifen, die den Transzendenzbezug universell und inhaltlich offen symbolisieren würden. Denn weder gibt es eine etablierte Tradition geteilter religiöser Räume oder einheitliche Vorstellungen der Religionen über das Verhältnis von Religion und Raum, noch haben sich in den verschiedenen Religionen ähnliche Zeichen und Symbole für die (Re-)Präsentation der Transzendenz herausgebildet. Im hier untersuchten Sample finden sich - im Unterschied zur in der Literatur aufgezeigten Vielfalt der Gestaltungsmöglichkeiten (Kraft, 2007)-lediglich zwei weitere Raum-Typen.

2. Räume christlicher Gastfreundschaft. Anders als in den als Kapellen ausgewiesenen Räumen in Fußballstadien wird in den Kirchen in der Sihlcity (Abb. 2) und am Bahnhof in Zürich, im Einkaufszentrum in Oberhausen sowie in den Räumen der Stille in den deutschen Bahnhofsmissionen die Offenheit gegenüber anderen Religionen betont. Dennoch ist die Konstellation von Dingen, Gerüchen und Symbolen an traditionelle christliche Raumarrangements angelehnt. Das Zentrum des Raumes bilden immer zentrale Medien christlichreligiöser Kommunikation: ein Kreuz und/oder ein mit Blumen und Kerzen geschmückter Tisch respektive Altar und/oder ein Lesepult (oft mit aufgeschlagener Bibel), die Bestuhlung ist auf sie ausgerichtet. Meist prägen auch bunte Glasfenster, edle Materialien und (indirekte) Lichtführung die Raumgestalt. Selten ist dagegen die Qibla, die Richtung Mekka weist, einfach auffindbar und oft fehlen gut sichtbare Symbole, Bilder und Kulturgegenstände nicht-christlicher Religionen oder es mangelt an Platz für Gebetsteppiche oder buddhistische Niederwerfungen. Zwar sind die entsprechenden Artefakte wie Gebetsteppiche, Meditationskissen oder der Qur'an vorhanden, jedoch meist auf marginale Plätze verwiesen und schwer zugänglich. Eine Gelegenheit für rituelle Waschungen oder zur Aufbewahrung von Schuhen, die im Islam und Hinduismus zentral sind, ist in keinem der untersuchten Räume vorhanden. Insgesamt entstehen so Räume, deren Atmosphären vor allem für diejenigen ansprechend sind, die sich in christlichen Kirchen wohlfühlen. Auch die angebotenen Andachten werden von Geistlichen durchgeführt, die - ebenso wie die Ehrenamtlichen - ausschließlich einer christlichen Kirche angehören. In keinem der be- 
suchten Räume ist die deklarierte Offenheit für andere Religionen mithin in der Raumgestalt und -nutzung angemessen umgesetzt. Die christlichen Kirchen gewähren den nicht-christlichen Religionen in diesem Raumtyp lediglich das Gastrecht - die Gäste müssen sich jedoch in die bestehende Raumorganisation einfügen. Vereinzelt beten Muslime in diesen Räumen, da sie hier ihrer Pflicht zum mehrmaligen täglichen Gebet nachkommen können; Juden sowie Hindus sind - nach Aussage der befragten Seelsorger - jedoch so gut wie nie in den Räumen anzutreffen.

3. neutrale Räume der Stille: Grundsätzlich anders sind die Räume der Stille (Einkaufspassage Leipzig ${ }^{12}$ (Abb. 3), Hamburger Hauptbahnhof) gestaltet, in denen versucht wird, durch eine „Architektur der Stille“ eine weltanschaulich neutrale Atmosphäre zu schaffen und dennoch auf Transzendenz zu verweisen. Diese Räume sind meist deutlich sparsamer eingerichtet, religiöse Symbole fehlen und das Zentrum markieren (vermeintlich) universelle Zeichen wie Kerzen, Blumen oder ein abstraktes Kunstwerk, denn abstrakter Kunst wird attestiert, Transzendenz sicht- oder erahnbar zu machen, ohne sie inhaltlich zu fixieren. Auch auf fixe Bestuhlung wird verzichtet, stattdessen erlauben Hocker, flexible Stühle oder Meditationskissen eine individuelle Platzwahl und so eine individuelle Ausrichtung im Raum. Andrew Crompton (2013) bezeichnet diese Raumgestaltung als „,negativen Typs“, da er die Nutzung des Raumes durch verschiedene Religionen mittels Exklusion religiöser Symbole zu erreichen sucht. Ob damit wirklich eine religionsübergreifende Offenheit erreicht werden kann, muss bezweifelt werden, denn inwieweit die Räume religiös unbestimmt und zugleich als religiös wahrgenommen werden können, bleibt ebenso fraglich wie die Inklusionswirkung der Ästhetik. Denn auch die vermeintlich neutrale Raumgestaltung ${ }^{13}$ mit ihrem Fokus auf ,architektonische Stille' sowie die Ausstattung mit abstrakter Kunst oder Meditationshockern folgen westlichen Sehgewohnheiten ${ }^{14}$ und orientieren sich an ,kunstaffinen Milieus“ (Beinhauer-Köhler, 2015:63). Auch die Ausrichtung auf eher individualisierte, körper-

\footnotetext{
${ }^{12}$ Der Raum der Stille in der Einkaufspassage Leipzig wurde am 13.4.2017 u. a. aufgrund der stark gestiegenen Mietpreise geschlossen.

${ }^{13}$ Die Vorstellung, einen Raum religiös neutral zu gestalten, erweist sich, wie Gilliat-Ray betont, als illusorisch, denn ,,behind every decision to place a table here (with or without a cloth over it), a chair there, or a picture on the wall (even if simply a pleasant landscape), an environment is inevitably ,materialising ' and, with it, the particular preferences of individuals with conscious or unconscious interests" (Gilliat-Ray, 2005:302).

${ }^{14} \mathrm{Zu}$ dem Schluss, dass die multireligiösen Räume in Deutschland nur bedingt ein integratives Potential entfalten, kommt auch Noss (2016)
}

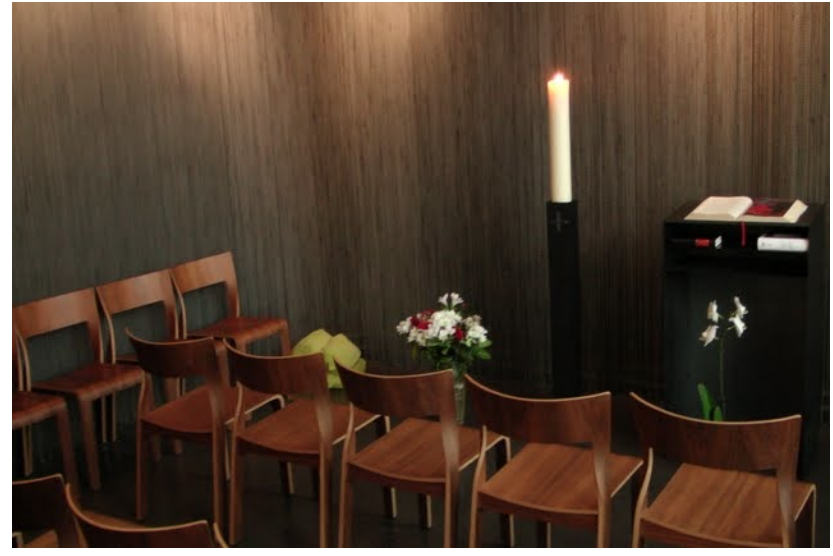

Abb. 2. Sihlcity-Kirche Zürich @Chris Hewson.

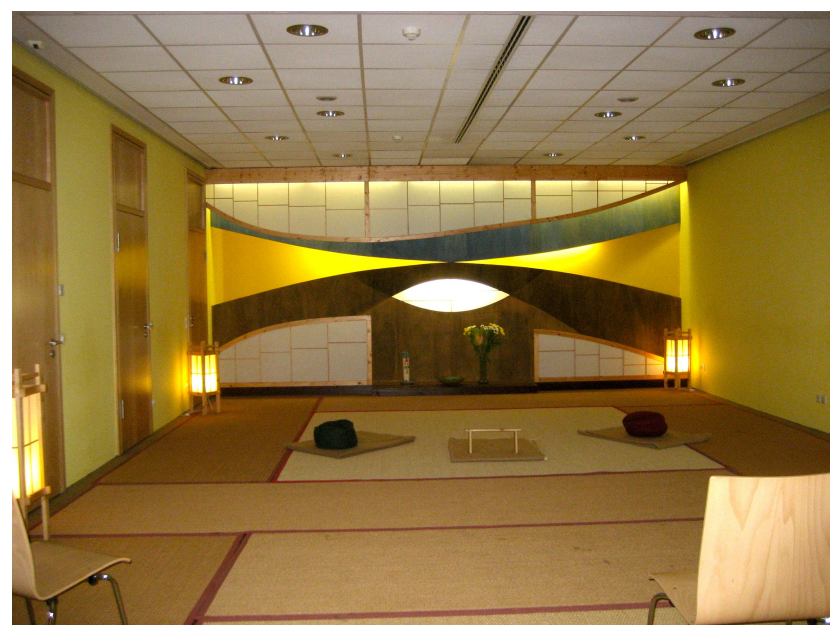

Abb. 3. Raum der Stille, Einkaufspassage Leipzig @Stefanie Duttweiler.

und bewegungslose, unsinnliche, rituelle Praktiken exkludiert so potentiell die (materialisierten) Artikulationen des Religiösen, die - wie beispielsweise im Hinduismus - grelle Farben, ,Kitsch“ und Düfte oder Musik und Bewegung einschließen.

Auffallend ist in diesem Sample der große Unterschied zu multireligiösen Räumen in Flughäfen oder Krankenhäusern. Diese sind oftmals Räume des „positiven Typs“ (Crompton, 2013), in denen Symbole und Gegenstände verschiedener Religionen relativ gleichwertig in einem Raum präsent sind, oder sie folgen Gestaltungslösungen, in denen Paraphernalien in Schränken oder Nischen aufbewahrt und nach Bedarf hervorgeholt werden können. Architektonisch wird so in den hier untersuchten Räumen das Problem umgangen, dass durch die Fülle von Gegenständen und Symbolen eine „in sich stimmige“ Raumgestalt und die religiöse Kenntlichkeit und Identifikation extrem erschwert wird. Sozial entfallen die indirekten Exklusionswirkungen 
der nicht-repräsentierten Religionen (Nagel, 2015:53) ebenso wie die z. T. weitreichenden Konflikte, die mit der multireligiösen Ausstattung und Ausrichtung einhergehen (Kong, 2010; Brand, 2012; Nagel, 2105, 2016).

Über die Gründe für diese Gestaltlösungen, die nichtchristliche Religionen kaum berücksichtigen, kann nur spekuliert werden. Die Interviews mit den Seelsorgern gaben allerdings Hinweise auf eine z. T. deutliche Abneigung der Kirchen gegenüber einem Raum für andere Religionen sowie auf die Kapitulation vor der Schwierigkeit, geeignete Kooperationspartner aus nicht-christlichen Religionen zu finden. Diese lediglich postulierte Offenheit bei architektonischer und sozialer Schließung zeigt aber auch, dass in den untersuchten Gebäudekomplexen kein Bedarf an multireligiösen Gebetsräumen unterstellt wird. Das überrascht - nicht nur angesichts der in Universitäten und Krankenhäusern eingeforderten Gebetsräume für Muslime, sondern auch angesichts der multireligiösen gesellschaftlichen Situation.

Das wirft jedoch auch die Frage auf, worin in einer multikulturellen und multireligiösen Gesellschaft der spezielle Bedarf für (christliche) religiöse Räume eigentlich besteht. Die tatsächliche Nutzung der Räume kann hier nur bedingt Auskunft geben; für die Frage nach dem Verhältnis von Religion und Gesellschaft scheint sie mir dennoch instruktiv. Denn es zeigt sich: Gut genutzt sind vor allem die Stadionkapellen als stimmungsvolle Orte für Taufe und Hochzeit sowie die kostenlosen Seelsorgegespräche in der Bahnhof- und der Sihlcity-Kirche in Zürich. Die in einigen Räumen angebotenen kurzen Andachten werden dagegen schlecht besucht; ${ }^{15}$ je nach Perspektive ließe sich das auch für die individuelle Nutzung der Räume (2016 durchschnittlich 10 Personen pro Tag in der Sihlcity-Kirche in Zürich) sagen. Dennoch wird den Räumen als Räumen großes Interesse entgegengebracht. So stoßen sie nach wie vor nicht nur auf wissenschaftliches, sondern auch auf (architektur-)touristisches Interesse; Teilnehmende sind meist Menschen mit christlicher Sozialisation mit und ohne Kirchenbindung. ${ }^{16}$ Darüber hinaus werden die Räume zur anschaulichen Religionsdidaktik genutzt. Da sie (christliche) Glaubensinhalte versinnbildlichen, werden in den meisten Räumen Schul- und Konfirmationsklassen mit religiöser Symbolik vertraut gemacht.

Die Nutzung der hier untersuchten Räume ähnelt mithin derjenigen herkömmlicher Kirchen - als Orte, Lebensübergänge zu feiern und der seelsorgerischen Lebensbegleitung

\footnotetext{
${ }^{15}$ Eine Nutzer-Statistik liege nur für die Sihlcity-Kirche vor. Hierin zeigt sich ein gravierender Rückgang der Besuchenden der Andacht (von 300 auf 60) sowie des Raumes der Stille (von 3300 auf 3000) und des Begegnungsraums (von 1500 auf 1000) zwischen 2014 und 2016, während die Zahl der Seelsorgegespräche von 550 auf 960 deutlich gestiegen ist (http://www.sihlcity-kirche.ch/portal/plugins/DPGportalKG/dl/ F-1492014236-6332edd8665bf4a3d7f6054d9a972c25.pdf).

${ }^{16} 2016$ wurden beispielsweise in der Sihlcity-Kirche in Zürich 530 Führungen durchgeführt. Doch auch alle anderen Geistlichen berichteten von zahlreichen Führungen.
}

sowie als Ziele kunsthistorischen oder gesellschaftlichen Interesses. Neue Zielgruppen oder neue Weisen der Spiritualität oder des interreligiösen Austausches eröffnen sich eher nicht.

\section{Fazit: Entschärfung der Säkularisierung - Zähmung des Religiösen}

Die spezifische Lage, ihre Zugänglichkeit, ihre Gestaltung und Nutzung macht diese neuen (multi-)religiösen Räume zu Orten, an denen paradigmatisch die Beziehung zwischen dem religiösen und säkularen Bereich sowohl implizit (in der architektonischen und organisatorischen Gestaltung) als auch explizit (in den Motiven der Entstehung sowie den Regelungen und Konflikten) verhandelt wird. In allen hier untersuchten Beispielen zeigt sich: Die räumlichen, symbolischen und rituellen Grenzen zwischen religiös und säkular werden durchlässig und Religion wird - auch in ihrer Pluralität - im säkularen Kontext Platz eingeräumt. Dieser Platz ist jedoch stark reglementiert und umkämpft: Als Mieter haben sich die Religionsgemeinschaften den zugewiesenen Platzierungen und Zugangsregeln des Kontextes anzupassen - und werden nicht selten vom Kontext räumlich und gestalterisch „geschluckt“. Doch die religiösen Räume werden in einigen Kontexten nicht lediglich in der eigenen ,Mitte“ geduldet, sondern sind willkommener Teil ihrer Corporate Identity. Diese hohe Wertschätzung, die den religiösen Räumen von Seiten des sie umgebenden Kontextes entgegengebracht wird, zeugt davon, dass den religiösen Räumen nicht zuletzt eine wichtige symbolische Funktion zugeschrieben wird. So gilt beispielsweise die Kapelle in Berlin als „Schmuckkästchen“ des Olympiastadions und für die Veltinsarena konstatiert der dort tätige evangelische Pfarrer: „Es ist ein gewisser Stolz da, dass man das hat. [...] Es ist ja auch eine gewisse Veredelung, wenn jetzt sogar Kirche hier ist. Schon im Unterbewusstsein, das ist eine andere Dimension, was Heiliges“ (Interview Filthaus). Durch die Integration eines religiösen Raumes und dem atmosphärischen, leiblich-affektiv erlebbaren Verweis auf eine ,,andere Dimension“ wird die Funktionslogik des säkularen Kontextes aufgebrochen und überschritten und so gewissermaßen ,veredelt“. Darüber hinaus wird durch die Einbettung eines religiösen Raumes in den Gebäudekomplex das Ansinnen von Shopping-Malls und Erlebnisbahnhöfen, eine „Stadt in der Stadt“ zu sein, komplettiert, denn religiöse Räume ,stehen ' auch symbolisch für die Mitte der Stadt und deren Werte (Duttweiler, 2011). ,Jetzt“", so kommentiert eine Besucherin die Eröffnung der SihlcityKirche in Zürich, ,ist es ein richtiges Dorf“ (Duttweiler, 2012b).

Beide Befunde zeigen - ebenso wie die Führungen, die eine gewisse Musealisierung des Religiösen implizieren dass der religiösen Dimension auch im säkularen Kontext eine wichtige Rolle zukommt. Mehr noch: Die religiösen Räume zeugen davon, dass symbolisch und räumlich ,die Kirche 
im Dorfe bleibt“", d. h., dass die neoliberale Umstrukturierung nicht vollständig gegen traditionelle Wertorientierungen opponiert. Zugespitzt lässt sich daraus folgern: Religiösen Räumen in nicht-religiösen Kontexten kommt die Funktion zu, die Folgen der Säkularisierung, keine sozialintegrierende ,Mitte‘ anbieten zu können, zu „entschärfen“.

Wie der Blick auf die innere Gestaltung der multireligiösen Räume (Typ 2 und 3) gezeigt hat, geschieht dies durch eine ambivalente Gleichzeitigkeit von deklarierter Offenheit gegenüber anderen Religionen und der architektonischen und sozialen Schließung ihnen gegenüber. So werden in den Räumen, in denen religiöse Neutralität angestrebt wird, die Besonderheiten jeder Religion negiert. Und auch in den Räumen, in denen die fünf Weltreligionen symbolisch adressiert werden, ${ }^{17}$ werden sie jedoch gestalterisch in keinem der Räume (angemessen) repräsentiert, da die Räume entweder ausschließlich als christliche Räume ausgewiesen sind oder in Gestaltung und Atmosphäre eindeutig darauf verweisen. Letzteres zeugt von einer gastgebenden und somit (ungewollt?) paternalistischen Haltung, die den nicht-christlichen Religionen gerade keinen eigenständigen Platz einräumt.

Das aus anderen Studien über multireligiöse Räume bekannte Phänomen, dem artikulierten Bedarf in Universitäten oder Krankenhäusern nach einem muslimischen Gebetsraum mit dem Errichten multireligiöser Räume zu begegnen (Nagel, 2105, 2016), kann für das hier untersuchte Sample nicht bestätigt werden, denn in keinem der befragten Kontexte wurde ein solcher Bedarf artikuliert oder - und das ist ebenso wahrscheinlich - er wurde nicht erhoben und/oder gehört. Doch auch in den hier untersuchten Räumen wird Muslimen der Raum für eine eigenständige Repräsentation und Praktik ihrer Religion verweigert. Sowohl in den Räumen der Stille als auch in den Räumen christlicher Gastfreundschaft werden die Inkompatibilitäten der verschiedenen Religionen, die - wie zum Beispiel die Geschlechtertrennung in islamischen Gebetsräumen - Anstoß erregen könnten, durch die architektonische Gestaltung „,gezähmt“. Diese „Zähmung“ der Religionen durch das Eingehen in eine (vermeintlich) gemeinsam geteilte Sphäre des Religiösen zeigt sich auch im Fokus auf individuelle Spiritualität, die den Wahrheitsanspruch der einzelnen Religionen sowie die Besonderheiten ihrer Dogmatik, Liturgie und Glaubenspraxis radikal reduziert. Diese verräumlichte, ,,bestimmte Unbestimmtheit“ des Religiösen scheint attraktiv, denn sie bietet die Möglichkeit, einen religiösen Raum zu etablieren, in ihn „einzutauchen“ und sich von seiner Atmosphäre faszinieren oder berühren zu lassen, ohne sich zwingend mit dem Inhalt der repräsentierten Religion(en) oder Weltanschauungen auseinandersetzen zu müssen. Dass diese Möglichkeit nur bedingt genutzt wird, spricht nicht gegen diese Attraktivität. Neben der sym-

\footnotetext{
${ }^{17}$ Alle mir bekannten Fälle - auch über das hier untersuchte Sample hinaus - unterscheiden sich von Räumen im angelsächsischen Raum, wo beispielsweise auch Sikhs, Bahai, Neopagane oder der Wicca-Religion Raum geboten wird.
}

bolischen Funktion ist es auch eine wichtige Funktion dieser Räume, im Not- und Bedarfsfall buchstäblich „,bereitzustehen" und die Option auf spirituelle Erfahrung und/oder einen Freiraum zu eröffnen.

Fasst man die aufgeführten Befunde zusammen, zeigt sich: Die neu entstehenden multireligiösen Räume sind ein Beleg dafür, dass Religion durchaus weiterhin ihren Platz und ihre Funktion in der und für die Gesellschaft hat - auch wenn sie ihre Selbstverständlichkeit eingebüßt hat und mit verschiedenen Herausforderungen durch eine sich verändernde Gesellschaft konfrontiert ist. In den hier vorgestellten doppelt pluralen Raumarrangements materialisieren sich diese Tendenzen und Herausforderungen und werden zugleich produktiv bearbeitet. Die gestalteten Antworten, die auf diese Herausforderungen gefunden werden, bearbeiten diese Fragen auf spezifische Weise - sie „zähmen“ die Ansprüche des Religiösen und entschärfen zugleich das Säkulare: Die Räume sind zentral platziert, ohne zugänglich zu sein; die Differenz zwischen sakral und profan wird inszeniert, ohne dass das Sakrale immer religiös gedeutet werden müsste; die Räume werden interessiert rezipiert, ohne dass sie intensiv genutzt würden; den nicht-christlichen Religionen und neuer Spiritualität wird zumindest in symbolischer Hinsicht Platz eingeräumt, meist allerdings ohne deren Besonderheiten gerecht zu werden, und die ,andere Dimension“ wird als Verweis auf eine „bestimmte Unbestimmtheit“ in Anspruch genommen, um den säkularen Gebäudekomplex zu ,,veredeln“.

Mir scheint hier etwas Paradigmatisches für das aktuelle, sich transformierende Verhältnis von Religion und Gesellschaft sichtbar zu werden. Denn die religiösen Räume erweisen sich sowohl im Hinblick auf ihre symbolischen Funktionen als auch ihre mögliche Nutzung vor allem als eine Sinnressource, die aufgrund ihrer semantischen Offenheit für viele anschlussfähig ist und aufgrund ihrer räumlichen Dimension sowohl sicht- als auch eigenleiblich erfahrbar werden kann. Dabei entspezifizieren sie sowohl die Charakteristika der Säkularisierung als auch die der Religion - ohne auf die Unterscheidung ganz zu verzichten. Durch diese materialisierte „Bearbeitung“ des Verhältnisses von Religion und Gesellschaft sind diese neuen religiösen Räume nicht nur ein Signum der Pluralisierung, Subjektivierung und Spiritualisierung des Religiösen, sie zeugen auch von der postsäkularen Gestalt und Gestaltung des Sozialen.

Datenverfügbarkeit. Die Datengrundlage kann bei der Autorin angefordert werden.

Interessenkonflikt. Die Autorin erklärt, dass kein Interessenkonflikt besteht.

Danksagung. Ich danke den anonymen Gutachtenden für ihre instruktive Kritik. 
Edited by: Benedikt Korf

Reviewed by: two anonymous referees

\section{Literatur}

Baumann, M. und Tunger-Zanetti, A.: Migration und religiöse Bauten - zur Neuaushandlung des öffentlichen Raums, Kunst und Kirche, 4, 32-39, 2008.

Beaumont, J. and Cloke, P.: Faith-Based Organisations and Exclusion in European Cities, Chicago University Press, Chicago, 2012.

Beckford, J.: Public Religions and the Postsecular. Critical Reflections, J. Sci. Stud. Relig., 51, 1-19, 2012.

Beinhauer-Köhler, B.: Im Zwischenraum. Plurale Raumarrangements aus religionswissenschaftlicher Perspektive, 55-76, 2015.

Beinhauer-Köhler, B., Roth, M. und Schwarz-Boenneke, B.: Viele Religionen - ein Raum?! Analysen, Diskussionen und Konzepte, Frank \& Timme, Berlin, 2015.

Bergmann, S. (Hrsg.): Theology in Built Environments, Transaction Publishers, New Brunswick/London, 131-150, 2012.

Brand, R.: Case Study 4: Multi-faith spaces as symptoms and agents of change, in: Religion and Change in Modern Britain, Herausgeber: Woodhead, L. and Catto, R., Routledge, Oxford und New York, 219-224, 2012.

Casanova, J.: Public religions in the modern world, University of Chicago Press, Chicago, 1994.

Crompton, A.: The architecture of multifaith spaces. God leaves the building, The Journal of Architecture, 18, 474-496, 2013.

Davie, G.: Religion in Britain since 1945. Believing Without Belonging, Blackwell, Oxford and Cambridge, 1994.

Delitz, H.: Architektursoziologie, Transcript, Bielefeld, 2009.

Díez de Velasco, F.: Multi-belief/Multi-faith Spaces: Theoretical Proposals for a Neutral and Operational Design, recode working paper series. online-working paper No. 26, online verfügbar unter: http://www.recode.info/wp-content/uploads/2014/01/ FINAL-26-D\%C3\%ADez-de-Velasco_fin.pdf, 2014.

Duttweiler, S.: Umnutzung von Kirchenräumen. Räume zwischen Religion und Politik, in: Kirchenbauten in der Gegenwart. Architektur zwischen Sakralität und sozialer Wirklichkeit, Herausgeber: Nollert, A., Volkenandt, M., Gollan, R.-M. und Frick, E. Hrsg., Regensburg, Pustet, 2011.

Duttweiler, S.: Sakrale Orte des Körperkults? Stadionkapellen zwischen Kirchenreligion und Ersatzreligion, in: Körper, Sport und Religion. Zur Soziologie religiöser Verkörperungen, Herausgeber: Gugutzer, R. und Böttcher, M., VS-Verlag, Wiesbaden, 193218, 2012a.

Duttweiler, S.: Jetzt ist es ein richtiges Dorf! Neue religiöse Räume an Orten (simulierter) Urbanität, in: Ausgesetzt. Exklusionsdynamiken und Exposureprozesse in der praktischen Theologie, Herausgeber: Pock, J., Hoyer, B. und Schüßler, M., Lit-Verlag, Münster, 277-294, 2012b.

Duttweiler, S.: Missionierende Räume? Neue religiöse Räume als Medien religiösen Wandels, in: Die Sinnlichkeit des Sozialen: Wahrnehmung und materielle Kultur, Herausgeber: Göbel, H. und Prinz, S., Transcript, Bielefeld, 195-218, 2015.

Duttweiler, S.: Grenzarbeit zwischen Sakralität und Profanität. Multireligiöse Räume in nicht-religiösen Kontexten, in: Architekturen und Artefakte. Zur Materialität des Religiösen, Herausgeber: Karstein, U. und Schmidt-Lux, T., 193-211, 2017.
Erne, T., Noss, P. und Bracht, C. (Hrsg.): Open Spaces. Räume religiöser und spiritueller Vielfalt, Jonas, Kromsdorf, 2016

Gieryn, T. F.: What Buildings Do, Theory and Society, 31, 35-74, 2002.

Gilliat-Ray, S.: From 'chapel' to 'prayer room'. The Production, Use, and Politics of Sacred Space in Public Institutions, Culture and Religion, An Interdisciplinary Journal, 5, 287-308, 2005.

Habermas, J.: Glauben und Wissen. Friedenspreisrede 2001, in: Zeitdiagnosen. Zwölf Essays 1980-2001, Suhrkamp, Frankfurt a.M., 249-262, 2003.

Hasse, J.: Was Räume mit uns machen - und wir mit ihnen. Kritische Phänomenologie des Raumes, Karl Alber, Freiburg, 2014.

Hervieu-Léger, D.: Die Rolle der Religion für die Herausbildung des sozialen Zusammenhalts in Europa, transit, 26, 101-119, 2003.

Holsappel-Brons, J.: Space for silence: The interplay between space and ritual in rooms of silence, in: Holy ground. Re-inventing ritual space in modern western culture, Herausgeber: Post, P. und Molendijk, A. L., Peeters, Leuven/Walpole, MA, 235-254, 2010.

Isenburg, W. und Sellmann, M.: Konsum als Religion? Über die Wiederverzauberung der Welt, Kühlen, Mönchengladbach, 2000.

Klein, C. und Schmidt-Lux, T.: Ist Fußball Religion? Theoretische Perspektiven und Forschungsbefund, in: Fußball. Fremdsprachen. Forschung, Herausgeber: Thaler, E., Shaker, Aschen, 18-35, 2006.

Knoblauch, H. und Schnettler, B.: Die Trägheit der Säkularisierung und die Trägheit des Glaubens. Der „Trendmonitor Religiöse Kommunikation 2003“ und die Kommunikation über Religion heute, in: Erosion des christlichen Glaubens? Umfragen, Hintergründe und Stellungnahmen zum ,Kulturverlust des Religiösen“, Herausgeber: Ziebertz, H.-G., Lit-Verlag, Münster, 5-14, 2004.

Kong, L.: Global shifts, theoretical shifts: Changing geographies of religion, Prog. Human Geogr., 34, 755-776, 2010.

Körs, A.: Gesellschaftliche Bedeutung von Kirchenräumen. Eine raumsoziologische Studie zur Besucherperspektive, VS-Verlag, Wiesbaden, 2012.

Kraft, S.: Räume der Stille, Jonas, Marburg, 2007.

Kunst und Kirche: Räume der Stille, 4, Ökumenische Zeitschrift für zeitgenössische Kunst und Architektur, Gütersloh: Gerd Mohn, 2004.

Kunst und Kirche: Multireligiöse Gebetsräume, 2, Ökumenische Zeitschrift für zeitgenössische Kunst und Architektur, Wien: Springer, 2010.

Löw, M.: Raumsoziologie, Suhrkamp, Frankfurt a.M., 2001.

Mennekes, F.: Sakralität der Leere, in: Kirchenbauten in der Gegenwart. Architektur zwischen Sakralität und sozialer Wirklichkeit, Herausgeber: Nollert, A., Volkenandt, M., Gollan, R.-M. und Frick, E., 236-243, 2011.

Moodie, D.: Research Report. Airport Chapels: Shifting from Denominational to Interfaith, Pluralism Project Newsletter, online verfügbar unter: http://pluralism.org/reports/view/82, 16 November 2005 .

Nagel, A.-K.: Kontaktzone oder Spannungsfeld? Multireligiöse Räume in religionssoziologischer Perspektive, Herausgeber: Beinhauer-Köhler, B., Roth, M. und Schwarz-Boenneke, B., 3554,2015

Nagel, A.-K.: Cui bono? Soziologische Einsichten zur Function und Nutzung multireligiöser Räume, Herausgeber: Erne, T., Noss, P. und Bracht, C., 61-71, 2016. 
Noss, P.: Inklusion, Exklusion und Differenz als theologische Kriterien für die Konzeption multireligiöser Räume, Herausgeber: Erne, T., Noss, P. und Bracht, C., 51-60, 2016.

Nynäs, P.: Spatiality, Practice and Meaning. The existential ambiguity of urban chapels, in: Theology in Built Environments, Herausgeber: Bergmann, S., Transaction Publishers, New Brunswick/London, 131-150, 2012.

Reese-Schäfer, W.: Von den Diagnosen der Moderne zu deren Überbietung. Die Postsäkularisierungsthese von Jürgen Habermas und der gemäßigte Postmodernismus bei Niklas Luhmann, Diskussionspapier Nr. 2010-2, urn:nbn:de:gbv:3:2-10570, 2010.

Schmitd-Lux, T.: Kirche und Aula zugleich? Eine Gebäudeinterpretation des Leipziger Paulinums, in: Architekturen und Artefakte. Zur Materialität des Religiösen, Herausgeber: Karstein, U. und Schmidt-Lux, T., 121-144, 2017.
Sorgo, G.: Kult, Konsum und Konvivalität: Einkaufen als Gottesdienst? in: Die Gretchenfrage. „Nun sag', wie hast du's mit der Religion?“, Herausgeber: Liessmann, K. P., Zsolnay, Wien, 212 235, 2008.

Van der Leeuw, G.: Religion in essence and manifestation. Princeton University Press, Princeton, 1986.

Wehrheim, J. (Hrsg.): Shopping Malls. Interdisziplinäre Betrachtungen eines neuen Raumtyps, VS-Verlag, Wiesbaden, 2007. 